\title{
Review Article \\ Effects of the Hormone Kisspeptin on Reproductive Hormone Release in Humans
}

\author{
Joanne L. Calley and Waljit S. Dhillo \\ Department of Investigative Medicine, Imperial College London, London W12 ONN, UK \\ Correspondence should be addressed to Waljit S. Dhillo; w.dhillo@imperial.ac.uk
}

Received 31 March 2014; Accepted 9 July 2014; Published 5 August 2014

Academic Editor: Paul Rösch

Copyright (C) 2014 J. L. Calley and W. S. Dhillo. This is an open access article distributed under the Creative Commons Attribution License, which permits unrestricted use, distribution, and reproduction in any medium, provided the original work is properly cited.

\begin{abstract}
The kisspeptins are a family of neuropeptides which act as upstream stimulators of gonadotrophin releasing hormone (GnRH) neurons. Kisspeptin signalling is prerequisite to establishing the normal human reproductive phenotype; loss of function mutations in the KISS1 or KISS1R gene produces normosmic hypogonadotrophic hypogonadism in humans and mice, whilst increased activation of KISSIR causes precocious puberty. Administration of exogenous kisspeptin to human subjects stimulates an acute gonadotrophin rise. Serum kisspeptin levels also markedly increase during pregnancy. The identification of kisspeptin has been one of the biggest discoveries in the field of reproductive endocrinology, since the isolation and sequencing of GnRH in 1977, and has generated a novel research avenue which has received much attention over the past decade. This research has delineated many properties of the KISS1-KISSIR system, but there is still further work to do. Understanding kisspeptin's role throughout our reproductive lifetime should help us better understand-and therefore treat-disorders of reproductive function. Promisingly, the current data supports the potential to develop kisspeptin based therapies. As an outlook article this paper focusses predominantly on our groups recent investigations into the effects of kisspeptin administration to humans and the potential therapeutic role of kisspeptin.
\end{abstract}

\section{Introduction}

1.1. Molecular Structure, Function, and Neuroanatomy of Kisspeptin. Kisspeptins are coded for by the KISS1 gene and act via binding with the G-protein coupled receptor 54 (GPR54) also known as the KISS1 receptor (KISS1R). The initial protein product of the KISS1 gene is a 145-aminoacid peptide, a member of the arginine phenylalanine (RF) amide group. It is cleaved into shorter, biologically active peptides known as kisspeptin-54, kisspeptin-14, kisspeptin13, and kisspeptin-10, where each number corresponds to the number of amino acids and with kisspeptin-10 representing the common C-terminal decapeptide sequence shared by all [1]. Kisspeptin expression has been identified in multiple tissues, including pancreas, adipose tissue, gonads, and placenta [1-3]; however, its main functional role is mediated by its expression within the central nervous system. Kisspeptin is produced by neurones in the infundibular (arcuate) nucleus of the hypothalamus. These neurons have been shown to have direct afferent connections onto GnRH neurons of the mediobasal hypothalamus in humans, and kisspeptin activity has been shown to induce upregulation of KISSIR within these GnRH neurons.

The effects of both kisspeptin-10 (KP-10) and kisspeptin54 (KP-54) have been investigated in animal studies [4] and both have been administered to humans [5-18]. Both peptides have been shown to be potent gonadotrophin secretagogues through activation of the hypothalamo-pituitarygonadal (HPG) axis at the hypothalamic level, with the gonadotrophin stimulatory effects of kisspeptin being abolished by preadministration of a GnRH antagonist [19]. Kisspeptin therefore regulates the activity of GnRH neurones, making it the top level of a final common pathway controlling reproduction.

1.2. Clinical Relevance. Disorders of reproduction which lead to reduced $\mathrm{GnRH}$ signalling result in hypogonadotrophic hypogonadism. They include idiopathic hypogonadotrophic hypogonadism (IHH) and hypothalamic amenorrhoea (HA). 
IHH occurs more frequently in males, is most often congenital, and has an estimated incidence between 1 and 10 of 100,000 live births [20, 21]. Functional hypothalamic amenorrhoea is a reversible form of hypogonadotrophic hypogonadism in females characterised by low LH pulse frequency, usually secondary to low body weight, weight loss, intensive exercise, or psychological stress and not due to any intrinsic abnormality of the hypothalamus or pituitary [21]. HA is a common disorder, accounting for a significant proportion of cases of secondary amenorrhoea. Increased stimulation of the HPG axis may lead to restoration of reproductive function in these patients. Overstimulation of the HPG axis produces downregulation of reproductive hormone secretion and is a therapeutic approach to treating prostate and breast malignancies as well as benign hormone dependent disorders such as benign prostate hyperplasia and endometriosis. In this review we discuss the effects of kisspeptin on reproductive hormone release. These findings have identified kisspeptin as a possible new therapeutic agent for the treatment of reproductive disorders in the future.

\section{The Discovery of Kisspeptin: Metastin and Genetic Mutations Affecting the KISS1-KISS1R System}

The KISS1 gene was identified in 1996 by Lee et al., a group from Hershey, Pennsylvania (hence, its unusual name, coined for the famous confectionary of this region, Hershey's kisses) [2]. Its G-protein coupled receptor and corresponding gene on Ch19p13 were discovered in 1999 [22], and the protein product of KISS1 was characterized in 2001 [3]. Kisspeptin was initially named metastin for its first studied role as a novel inhibitor of the metastatic activity of tumours [3]. Early literature on kisspeptin was fairly sparse and was focussed on its involvement in oncology pathways. Some clues that it might play a role in reproductive endocrinology were provided by the fact that KISS1R expression was localized to (amongst other tissues) placenta, pituitary and hypothalamus; that there was notably high expression of both kisspeptin and KISS1R in human placenta, with the initial isolation of kisspeptin from human placental extracts; and the fact that intravenous kisspeptin administration in rats was noted to stimulate oxytocin release [1]. At the beginning of 2003, the group from Japan who first isolated and sequenced kisspeptin [3] also developed the first kisspeptin polyclonal radioimmunoassay and published data demonstrating that kisspeptin levels were detectable in the plasma in human subjects. They also reported massive elevations in plasma kisspeptin during pregnancy, suggesting its role as a placentaderived hormone [23].

The interest in kisspeptin amongst the reproductive endocrinology community was only truly piqued following two publications in late 2003. These publications described deletions or inactivating mutations in the kisspeptin receptor gene $(K I S S 1 R)$ in individuals suffering from IHH [24, 25]. A seminal report from de Roux et al. [24] utilized genetic microarray and linkage analysis techniques to identify a large deletion in KISS1R on chromosome 19p13, leading to production of truncated and nonfunctional G-protein coupled transmembrane kisspeptin receptors in five individuals affected by $\mathrm{IHH}$ in a consanguineous family in France. Shortly following this Seminara et al. published data implicating two novel point mutations in the KISSIR gene in the aetiology of IHH. The first mutation was identified during investigation of a large Saudi Arabian family with multiple same generation sufferers of $\mathrm{IHH}$, who had previously through linkage analysis had the affected area mapped to a region of interest containing the KISSIR gene [25]. The pedigree was consistent with a pattern of autosomal recessive inheritance. Affected individuals in the family were found to be homozygous for a point mutation (L148S) in exon 3 of the KISS1R gene, which was not identified in any unaffected family members or any of 260 normal controls. DNA sequencing of several unrelated individuals with $\mathrm{IHH}$ led to the identification of a further KISS1R point mutation (R331X) in a single male proband which resulted in a premature stop codon.

Generation of KISSIR knockout mice confirmed the importance of this G-protein coupled receptor and its ligand kisspeptin, as key players in reproductive endocrinology [25, 26].

The genetic causes of both kisspeptin and KISS1R dysfunction have recently been extensively reviewed [27]. To date, there have been further null mutations discovered in patients with $\mathrm{IHH}$ in both the KISS1R [28] and recently the KISS1 gene itself [29], with genetic defects in the KISS1KISS1R system appearing to account for around $2 \%$ of all cases of $\mathrm{IHH}$ worldwide [30]. Additionally, activating mutations of KISS1R have been identified in individuals with central precocious puberty [31].

\section{Effects of Administration of Exogenous Kisspeptin to Humans}

These findings generated intense interest that kisspeptin may be useful therapeutically. Kisspeptin has now been administered to humans in both the KP-10 and KP-54 isoforms. All of the studies so far have shown it to be safe for use in humans with no demonstrable side effects. In keeping with the definition of an outlook article this paper focusses predominantly on our groups recent investigations into the effects of kisspeptin administration to humans. However, we also reference the important contributions from other leading investigators in this area to provide the reader with details of the work from our group in the context of other relevant published literatures.

3.1. Pharmacokinetics of the Kisspeptin Isoforms: Kisspeptin-10 and Kisspeptin-54. In contrast to a large body of animal work, only a small but select number of studies involving exogenous administration of kisspeptin have been carried out in humans [5-18]. All of the studies have used either the KP-10 or the KP54 isoform and various doses and routes of delivery have been investigated including single or multiple dosing regimens, intravenous or subcutaneous bolus, and intravenous infusion (IVI) of short and long duration. Doses explored in acute administration have ranged from a single intravenous bolus 
of $0.0077 \mathrm{nmol} / \mathrm{kg}$ [7] to a total dose of $72.3 \mathrm{nmol} / \mathrm{kg}$ over a $22.5 \mathrm{~h}$ infusion [7]. In all studies involving the administration of kisspeptin to humans, the natural, unmodified peptide sequence of KP-10 or KP-54 has been used.

KP-10 and KP-54 have almost identical biological activity and potency in vitro. However, when given in vivo to nonhuman mammals, KP-54 has been shown to have a slower onset and longer duration of action than KP-10. The half-life $\left(t_{1 / 2}\right)$ of both isoforms in healthy human volunteers have been calculated using the decay curve of kisspeptin IR; $t_{1 / 2}$ KP-54 was calculated in 2005 to be $27.6 \pm 1.1 \mathrm{~min}$ [5] and $t_{1 / 2} \mathrm{KP}$ 10 was demonstrated in 2011 to be around 4 minutes $(3.8 \pm$ $0.3 \mathrm{~min}$ (men), $4.1 \pm 0.4 \mathrm{~min}$ (follicular-phase women), and $4.1 \pm 0.4 \mathrm{~min}$ (preovulatory-phase women)) [8]. This suggests that there are differences in the pharmacokinetic handling of the two isoforms, which may translate into differences in the effect on reproductive hormone release when peripherally administered. The evidence for this from human studies will be explored further in the relevant sections below.

\subsection{Kisspeptin in Healthy Men}

3.2.1. Kisspeptin-54 Administration to Healthy Human Males. The first-in-man kisspeptin study was carried out by our group in 2005 using KP-54 [5]. This study was done in two parts, the first being a dose-response study in healthy male volunteers using a 90-minute infusion at rates in the range of $0.12-40 \mathrm{pmol} / \mathrm{kg} / \mathrm{min}$ for the first 30 minutes, with the rate being halved for the remaining $60 \mathrm{~min}$ of the infusion in order to achieve a steady state plasma concentration (giving a total dose of $0.0075-2.4 \mathrm{nmol} / \mathrm{kg}$ to each volunteer over the study period). These relatively short infusions demonstrated that a plasma-kisspeptin immunoreactivity (IR) greater than $300 \mathrm{pmol} / \mathrm{L}$ (achieved by infusing KP-54 for 90 minutes at an initial rate of $0.24 \mathrm{nmol} / \mathrm{kg} / \mathrm{h}$ ) results in near maximal $\mathrm{LH}$ release in healthy men, with a mean peak $\mathrm{LH}$ of $11.4 \pm$ $1.7 \mathrm{iU} / \mathrm{L}$ demonstrated in this setting. The second study arm thus used a single infusion at this rate of $0.24 \mathrm{nmol} / \mathrm{kg} / \mathrm{h}$ to compare the time course of events with that observed during a saline infusion control. Here, the kisspeptin infusion significantly increased plasma LH, FSH, and testosterone at 90 minutes compared with saline infusion (mean $90 \mathrm{~min} \mathrm{LH:}$ kisspeptin, $10.8 \pm 1.5$ versus saline, $4.2 \pm 0.5 \mathrm{U} /$ liter, $P<$ 0.001; mean 90 min FSH: kisspeptin, $3.9 \pm 0.7$ versus saline, $3.2 \pm 0.6 \mathrm{U} /$ liter, $P<0.001$; mean $180 \mathrm{~min}$ testosterone: kisspeptin, $24.9 \pm 1.7$ versus saline, $21.7 \pm 2.2 \mathrm{nmol} / \mathrm{liter}$, $P<0.001)$. As demonstrated consistently in all studies of acute administration of kisspeptin, a far more potent effect for stimulation of LH than FSH was seen, with testosterone levels being the least potently affected of all.

3.2.2. Kisspeptin-10 Administration to Healthy Human Males. KP-10 was first administered to healthy human males in 2011 [7]. The investigators demonstrated that giving KP-10 as bolus doses resulted in a significant increase in $\mathrm{LH}$ release and a smaller increase in FSH release. In addition a prolonged intravenous infusion of KP-10 increased both the amplitude and frequency of $\mathrm{LH}$ pulses during the infusion period in healthy men [7]. These publications clearly demonstrated that giving kisspeptin intravenously could reliably stimulate $\mathrm{LH}$ release in healthy men. A second study in 2011 [17] showed that intravenous administration of a bolus of KP-10 to healthy males resulted in resetting of the periodicity of endogenous LH pulse generation; a novel finding implicating kisspeptin as a key player in the control of the central GnRH clock. Our group has also investigated the effects of IV bolus KP10 in healthy males. In one arm of a study published in 2011 [8], IV bolus of KP-10 was administered to 10 healthy male participants at doses of $0.3,1.0,3.0$, and $10 \mathrm{nmol} / \mathrm{kg}$. By measuring plasma kisspeptin IR we were able to document the pharmacokinetic handling of an exogenous kisspeptin injection. It was observed that plasma kisspeptin IR peaked at 10 minutes after a bolus injection $10 \mathrm{nmol} / \mathrm{kg} \mathrm{KP}-10$ and returned to undetectable levels after 50 minutes. Plasma LH was significantly elevated following a KP-10 bolus injection at all concentrations investigated and was maximally elevated 30-40 minutes after injection with a return to baseline at 180 minutes. In contrast to the findings of the earlier study by George et al. [7] where a dose of $0.77 \mathrm{nmol} / \mathrm{kg}$ produced maximal peak $\mathrm{LH}$, when area under the curve (AUC) of LH was used as the measure of activity, maximal LH stimulation was seen after a bolus dose of $10 \mathrm{nmol} / \mathrm{kg}$ (AUC LH $6.1 \pm 1.3 \mathrm{~h} \cdot \mathrm{iU} / \mathrm{L}$ ). AUC FSH was also increased following bolus doses of 1.0 and $3.0 \mathrm{nmol} / \mathrm{kg}$ but was less robustly stimulated than $\mathrm{LH}$ and slower to reach peak levels which occurred at 45-150 minutes after injection. Serum testosterone levels showed a steady rise following the lower dose boluses of KP-10 ( 0.3 and $1.0 \mathrm{nmol} / \mathrm{kg})$; however, these elevations were marginal and not consistent throughout the dose range tested, leading the researchers to conclude that a single bolus dose at the range tested was not sufficient to produce downstream stimulation of sex steroid release within the studied timeframe.

In summary, both KP-10 and KP-54 acutely stimulate a gonadotrophin rise in healthy men and a first $\mathrm{LH}$ peak within 60 minutes of administration, with much more potency for stimulation of LH than FSH and a smaller effect on serum testosterone. If a bolus of kisspeptin is given there is evidence to suggest that the mechanism controlling the timing of release of endogenous $\mathrm{GnRH}$ pulses is reset, and if kisspeptin is given as a continuous infusion the number of LH pulses in a given period may increase.

3.3. Kisspeptin in Healthy Women. Quantifying the effects of kisspeptin on gonadotrophin release in women is a more challenging task because, unlike in men where the target organs of an adult patient will show a relatively fixed and reproducible response to the same dose of kisspeptin provided their baseline nutritional status and health remains stable, the response to kisspeptin varies markedly in women depending on their phase of the menstrual cycle.

The data collected so far suggests that kisspeptin stimulates gonadotrophin release in females most potently during the preovulatory phase of the menstrual cycle (15-16 days before next predicted period), followed by the luteal phase and then the follicular phase (day 2-10). There have been some differences in the data collected from the small number of studies of kisspeptin administration to healthy women 
so far, with some evidence to suggest that KP-10 has no effect if given during the follicular phase, and other evidences suggesting there is a retained but less potent dose-dependent response in this part of the menstrual cycle.

\subsubsection{Kisspeptin-54 Administration to Healthy Human Fe-} males. A study in 2007 from our group first investigated the effects of subcutaneous administration of KP-54 to human females [11]. 8 healthy female volunteers with regular menstrual cycles were selected to investigate the effects on LH following a subcutaneous bolus of KP-54. Initially a doseresponse study was carried out using various doses ranging from 0.2 to $6.4 \mathrm{nmol} / \mathrm{kg}$, with each dose being given to $3-$ 4 volunteers. Akin to the effect seen in healthy males given KP-54, a dose-dependent LH rise was exhibited in women in the follicular phase of the menstrual cycle on receipt of kisspeptin. Based on this, a dose of $0.4 \mathrm{nmol} / \mathrm{kg}$, which elicited only a small but demonstrable response, was selected to carry out a further double-blind placebo-controlled study of the effect of KP-54 in various stages of the menstrual cycle. KP-54 was found to stimulate an LH response when given at any point in the menstrual cycle; however, the intensity of the response showed great variation directly linked to the phase of the menstrual cycle, being greatest in the preovulatory phase, followed by the luteal phase and then the follicular phase. The effects of IV bolus KP-54 were examined in one arm of a study from our group which directly compared the effects of IV bolus kiss-54 and kiss-10 and is discussed in detail in the next section.

\subsubsection{Kisspeptin-10 Administration to Healthy Human Fe-} males. Research in healthy women using KP-10 was first carried out by our group in 2011. A multifaceted study of KP-10 was used to directly compare many variables of kisspeptin administration [8]. These included the effects of (i) gender-comparing IV bolus KP-10 in men versus women; (ii) kisspeptin isoform-comparing the effect of IV bolus KP10 versus KP-54 in females and; (iii) route-comparing the effect of IV bolus versus sc bolus versus 90 minute IV infusion (IVI) of KP-10 in women. Pharmacokinetic profiling of KP-10 during an intravenous infusion was also performed. By performing a direct comparison of female versus male subjects, the data confirmed the sexually dimorphic gonadotrophin response to kisspeptin in humans, reinforcing the finding that females are most responsive in the preovulatory phase of the cycle (mean AUC increase in LH $30.3 \pm 7.7 \mathrm{~h} \cdot \mathrm{iU} / \mathrm{L}$ in preovulatory phase women).

Interestingly, in this study, there was no demonstrable response detected on serial measurement of serum LH and FSH following KP-10 administration by any of the routes used at doses up to $10 \mathrm{nmol} / \mathrm{kg}$ via IV bolus, $32 \mathrm{nmol} / \mathrm{kg} \mathrm{sc}$ bolus, and $720 \mathrm{pmol} / \mathrm{kg} / \mathrm{min}$ IVI (total dose $64.8 \mathrm{nmol} / \mathrm{kg}$ ) when given to women in the follicular phase of the cycle. This was despite similar rises in plasma kisspeptin IR levels observed in men who were given the same dose and who exhibited significant rise in $\mathrm{LH}$ from baseline at all tested doses (maximal stimulation in men given KP-10 occurred at $10 \mathrm{nmol} / \mathrm{L}$, mean AUC LH increase $6.1 \pm 1.3 \mathrm{~h} \cdot \mathrm{iU} / \mathrm{L}$ ). By contrast, following an IV bolus of $1 \mathrm{nmol} / \mathrm{kg} \mathrm{KP}-54$ to women in the follicular phase, gonadotrophin secretion was robustly stimulated (mean AUC LH increase $27.0 \pm 11.8 \mathrm{~h} \cdot \mathrm{iU} / \mathrm{L}$ ). This suggested there may be a different response to the KP-54 and KP-10 isoforms by women in the follicular phase. Two other studies in females have, however, had different results that show some effects on gonadotrophin levels following KP-10 administration in the follicular phase in healthy females.

A study by Chan et al. in 2012 investigated the effects of IV bolus KP-10 $0.24 \mathrm{nmol} / \mathrm{kg}$ in healthy women [9]. Half of the women were unresponsive to KP-10 in the early follicular phase; however, five of the ten women tested showed a significant though small gonadotrophin response to $0.72 \mathrm{nmol} / \mathrm{kg}$ IV bolus of KP-10 [9].

Another study from 2012 which further investigated the role of sex steroids as direct modulators of the responsiveness of GnRH neurons to KP-10 showed that after an IV $0.3 \mathrm{mcg} / \mathrm{kg}$ bolus of KP-10 was delivered to women in in the early follicular phase a change in AUC LH of $2.3 \pm 50.8 \mathrm{IU} / \mathrm{lh}$ occurred. Peak LH rose from $6.3 \pm 1.2$ at the baseline to a peak of $9.4 \pm 1.3 \mathrm{IU} / \mathrm{L}(P=0.006)$ at 30 minutes [14]. To investigate further how kisspeptin responsiveness was related to background sex steroid milieu, they elected to compare the response to IV KP-10 of this group of women in the follicular phase of the menstrual cycle with a group of postmenopausal women (low sex steroid/high gonadotrophin) and with a group of premenopausal women taking a combined oestrogen-progesterone oral contraceptive pill (OCP) (high sex steroid/low gonadotrophin) and a group using a progesterone only implant contraceptive. They demonstrated that states of high background oestrogen (combined OCP) but not high progesterone only (progesterone only implant device) suppressed the LH response to kisspeptin and that the LH response to kisspeptin appeared to be enhanced in a sex steroid deficient milieu [14].

3.4. Relative Potency of Kisspeptin in the Sexes. Some of the research described above enables us to directly compare the effects of kisspeptin in men and women; unfortunately we can only make a true comparison of the effects of KP-10 in men versus women and not KP-54, as only KP-10 has been given to men and women using the same protocol (dose, route, and method of administration) and only the IV bolus route has been directly compared. This was done first by Jayasena et al. [8] and subsequently by Chan et al. who utilized an identical protocol in their paper of KP-10 administration to men in 2011 [17] as used in their 2012 study in women in various phases of the menstrual cycle [9] and by George et al. who have studied the effects of an IV bolus $0.3 \mathrm{mcg} / \mathrm{kg} \mathrm{KP}-10$ in both men and women [7, 14]. All groups have used AUC as their measure of response. Chan et al. used a single dose of $0.24 \mathrm{nmol} / \mathrm{kg}$; Jayasena et al. studies the doses $0.3,1.0,3.0$, and $10.0 \mathrm{nmol} / \mathrm{kg}$.

Broadly, the groups have demonstrated the following overriding principles in response to an IV bolus of KP-10.

(i) Kisspeptin rapidly induces release of a single pulse of $\mathrm{LH}$ in men, which is generally of greater amplitude than endogenous pulses.

(ii) LH release is robustly stimulated by a single bolus of IV kisspeptin given to women in the preovulatory 
phase [9]. The time frame to peak is similar to that observed in men (40 minutes) [9]. LH stimulation is much larger in amplitude in the preovulatory phase than in any other phase in women, but a similar fold rise in $\mathrm{LH}$ release is seen in women in the preovulatory and midluteal phase and men (around 2-fold increase above endogenous activity) when analysed in light of baseline LH.

(iii) Women in the follicular phase of the menstrual cycle show the lowest inducible response to KP-10 and cannot be reliably stimulated by KP-10 administration up to relatively high doses.

It is therefore clear that the KISS1-KISS1R system is profoundly influenced by the circulating sex steroid milieu, in agreement with patterns of kisspeptin regulation by endogenous sex steroids previously demonstrated in animal species $[32,33]$.

3.5. Kisspeptin in Individuals with Disorders of Reproduction. The effects of kisspeptin have been investigated in women with hypothalamic amenorrhoea and in individuals with IHH secondary to a mutation of the TAC3 or TACR3 gene.

3.5.1. Kisspeptin in IHH Secondary to Mutations of TAC3 or TACR3. TAC3 and TACR3 code for the tachykinin neurokinin $\mathrm{B}(\mathrm{NKB})$ and its receptor NKR3, respectively [15]. The NKB-NKR3 signalling pathway is also a hypothalamic signalling pathway, and although NKB is not a potent inducer of gonadotrophin release in the same way that kisspeptin is, this pathway is known to have essential permissive properties with regard to hypothalamic stimulation of the HPG axis, such that those with absence of NKB signalling exhibit a phenotype almost identical to the $\mathrm{IHH}$ seen in patients with loss of function mutations in KISS1 or KISS1R [34]. The relationship between kisspeptin and NKB is discussed further in a subsequent section of this review.

In two young men with homozygous mutations in c.209$1 G_{-} C$ of the TAC3 gene, one young man with a c.738-1G_A homozygous mutation of the TACR3 gene, and one young woman with a homozygous deletion of TACR3, continuous infusion of KP-10 at a rate of $1.5 \mathrm{mcg} / \mathrm{kg} / \mathrm{h}$ for $12 \mathrm{~h}$ on two consecutive days has been used to reinstate gonadotrophin pulsatility, with significant elevations of LH and FSH levels in all participants [15]. This demonstrates that although kisspeptin and NKB both appear necessary for puberty and reproductive function in humans, kisspeptin is able to stimulate effective $\mathrm{GnRH}$ release in the absence of $\mathrm{NKB}$ when given exogenously.

3.5.2. Kisspeptin in Women with Hypothalamic Amenorrhoea. Intermittent administration of kisspeptin to women with HA with the aim of restoring reproductive function has been attempted with two different protocols, both utilising subcutaneous KP-54. Participants with HA are acutely responsive to subcutaneous kisspeptin but do not necessarily sustain this response with ongoing administration. More recently, our group has outlined the response of women with HA to a continuous low dose intravenous infusion of kisspeptin. The outcome of these investigations is discussed below in the context of kisspeptin's ability to induce tachyphylaxis or downregulation of the HPG axis following an initial stimulation phase.

3.6. Kisspeptin and the Phenomenon of Tachyphylaxis. The phenomenon of tachyphylaxis is well described in mammalian endocrinology. It is a notable property of $\mathrm{GnRH}$ receptors when they are stimulated at supraphysiological levels. Hence, medical castration can be induced therapeutically with the use of high potency $\mathrm{GnRH}$ analogs for the treatment of prostate cancer [35].

KP-10 has been shown to cause tachyphylaxis when administered as a high dose continuous intravenous infusion to male rhesus monkeys, with an initial acute stimulation of gonadotrophin release lasting only 3 hours, beyond which there is a rapid drop to baseline levels $[36,37]$. This response is also seen in rats who demonstrate tachyphylaxis during infusions persisting more than 24 hours [38]. Tachyphylaxis following an initial upregulation of LH secretion has previously been described in rodents given a continuous subcutaneous infusion of kisspeptin beyond day 2 of administration [39]. When given as intermittent injections, however, chronic administration of KP-10 can induce chronic stimulation of the HPG axis, as seen in both juvenile female rats and juvenile male monkeys, in which twice-daily injections for $5 \mathrm{~d}$ and hourly injections for $2 \mathrm{~d}$, respectively, produce animal models of precocious puberty $[40,41]$. Additionally, in a rat model of hypothalamic amenorrhoea, reproductive function can be rescued with twice daily supplementation of KP-10 [42]. It seems that mammals may react anywhere on the spectrum of stimulation to suppression of the HPG axis in response to chronic kisspeptin, with potential factors determining this response being the dose and method of kisspeptin delivery and the species and age of the study subject.

The longest continuous intravenous infusion of kisspeptin that has been investigated in humans was the $22.5 \mathrm{~h}$ infusion study conducted by George et al. [7]. No evidence of tachyphylaxis was seen in healthy males over the study period at the two doses studied.

Perhaps of greater value with a view to manipulation of kisspeptin for a therapeutic purpose is to examine the effects of intermittent administration of kisspeptin over a prolonged period time, and to quantify this not only in healthy individuals but also in a potential treatment group (the response to kisspeptin is profoundly altered by the circulating sex steroid milieu and could therefore be predicted to be very different in people affected by reproductive disorders such as $\mathrm{IHH}$ or HA).

The effects of administration of kisspeptin intermittently for periods greater than 24 hours to humans has to date been studied in both healthy women and women with functional hypothalamic amenorrhoea (HA), with some interesting and disparate results [16].

In the first of these studies, females with HA, a condition characterized by low levels of background $\mathrm{GnRH}$ pulse generation, were given twice daily subcutaneous injections of $6.4 \mathrm{nmol} / \mathrm{kg} \mathrm{KP-54}$ for two weeks [12]. This was a 
single-blinded study using saline injections as a control. This method of administration resulted in tachyphylaxis. Acute administration of KP-54 stimulated a potent increase in serum LH of over 20-fold with serum LH rising to $24.0 \pm$ 3.5 IIU/L from baseline and FSH 9.1 $\pm 2.5 \mathrm{IIU} / \mathrm{L}$ from baseline. Downregulation became evident at just 24 hours following KP-54 injections [12]. The mechanism by which this tachyphylaxis occurred was further investigated via administration of a GnRH test (100 mcg IV GnRH bolus) on the final day of the study. Interestingly, females with HA who no longer responded to KP-54 retained responsiveness to GnRH, indicating that the desensitization effect was occurring at the hypothalamic level, most likely at the level of the KISS1R [12].

Healthy females do not however seem to be as prone to tachyphylaxis. When an identical protocol was applied to healthy female volunteers in the follicular phase of their menstrual cycle, menstrual cyclicity was preserved with minimal downregulation of gonadotrophins [16]. This difference in response to chronic kisspeptin may be a result of differences in KISS1R sensitivity or expression in the hypothalamus of healthy women versus women with HA. This study of chronic kisspeptin administration also demonstrated that persistent administration in the first half of the menstrual cycle in healthy females may hasten follicular maturation. Compared to those receiving saline control, these subjects experienced an earlier onset of both menses and the serum progesterone rise to $\geq 21 \mathrm{mmol} / \mathrm{L}$ by approximately 48 hours.

This raises the possibility that kisspeptin may be a viable agent for use as a treatment in anovulatory causes of infertility, or potentially as an ovulation trigger in women undergoing treatment for infertility that requires controlled timing for example in vitro fertilisation.

In light of the highly sensitive response to kisspeptin seen in women with HA [12], a further study attempted to stimulate the sensitized GnRH neurones with a more gentle protocol by extending the interval between kisspeptin doses and using a protocol of twice weekly subcutaneous KP-54 $(6.4 \mathrm{nmol} / \mathrm{kg})$ [6]. This was continued for a total of 8 weeks. During this period a pattern of initial, partial desensitization of the gonadotrophin response was observed, followed by a plateau response, with $\mathrm{HA}$ patients remaining partially responsive to KP-54 during this dosing regimen from day 14 until the end of the 8-week study period.

This data suggests that kisspeptin may be useful in the treatment of HA in humans; however, it must be noted that in both studies investigating the effects of KP-54 in women with hypothalamic amenorrhoea, even in light of significantly raised levels of gonadotrophins using the twice weekly protocol, changes were not reflected at the ovarian level; that is, no woman demonstrated any significant follicular growth on serial ultrasound scanning. There is a need to better understand the reasons for this. One plausible explanation may be the differential potency of kisspeptin for $\mathrm{LH}$ and FSH, with kisspeptin evoking lesser stimulation and quicker desensitization of the FSH response and therefore leading to a relative lack of FSH, the essential hormone controlling follicle maturation.

Further work has recently been carried out by our group to determine if there is a dose range at which continuous intravenous infusion of $\mathrm{KP}-54$ is able to restore $\mathrm{LH}$ pulsatility in females with HA without inducing tachyphylaxis [43]. A single-blinded continuous IV infusion of either gelofusine vehicle or KP-54 at a rate of $0.01,0.03,0.10,0.30$, or $1.00 \mathrm{nmol} / \mathrm{kg} / \mathrm{h}$ (rate doubled for the first 30 minutes to achieve a steady state plasma level) was administered to 5 female volunteers with HA for a minimum of $8 \mathrm{~h}$ with regular serum sampling and measurement of gonadotrophin and sex steroid levels. Kisspeptin IR analysis confirmed that serum $\mathrm{KP}-54$ levels in these patients reached a plateau level at $1 \mathrm{~h}$ into the infusion and remained constant until termination of the infusion. The number of LH pulses, secretory mass of each $\mathrm{LH}$ pulse, and estimate levels of basal and pulsatile LH secretion were recorded at each study visit. The results demonstrate that continuous low-dose KP-54 IV infusion can restore baseline LH secretion and LH pulsatility in women with HA, with evidence in support of the existence of a dose-dependent range, or therapeutic window, in which this occurs.

Within our small sample size $(n=5)$ there was individual variation in responsiveness to KP-54, but at the optimal dose for each patient within the range studied a KP-54 infusion increased the number of $\mathrm{LH}$ pulses recorded during the 8-hour-infusion period by an average of threefold (mean baseline number of $\mathrm{LH}$ pulses per $8 \mathrm{~h}$ : $1.6 \pm 0.4$, vehicle; $5.0 \pm 0.5, \mathrm{KP}-54, P<0.01$ versus vehicle), with a mean peak LH pulse secretory mass 6 -fold higher when compared with vehicle.

Desensitization to the effect of KP-54 was observed during the highest infusion rate of $1.0 \mathrm{nmol} / \mathrm{kg} / \mathrm{h} \mathrm{KP}-54$. Serum $\mathrm{LH}$ levels peaked at $5 \mathrm{~h}$ and then gradually declined until the infusion was ended at $10 \mathrm{~h}$. Patients retained responsiveness to a GnRH test at the end of the infusion and one week later, which suggests, in agreement with findings from an earlier study by our group [12] that this desensitization does not involve the pituitary gland and is likely to occur at the hypothalamus.

3.7. Kisspeptin Effects on LH Pulse Generation-Kisspeptin Reset the Biological Clock in Men but Not Women. We still understand relatively little about how the biological clock that determines the release of $\mathrm{GnRH}$ in a pulsatile fashion is controlled [44]. The anatomical region thought to contain endogenous GnRH pulse pacemaker activity is the mediobasal hypothalamus, which contains GnRH neurons and closely related neurons, inclusive of afferent kisspeptin connections [45]. Due to the regulatory role of kisspeptin on $\mathrm{GnRH}$ neurones, it is thought that kisspeptin may play a major role in pulse generation. In rats, administration of a kisspeptin antagonist directly into the arcuate nucleus caused a profound reduction in the number of $\mathrm{LH}$ pulses generated [46]. Neurokinin B has also been heavily implicated in control of GnRH pulse generation, as TAC3 and TACR3 mutants have been shown to develop hypogonadotrophic hypogonadism through reduction in the number of $\mathrm{GnRH}$ pulses released [47].

Administration of kisspeptin may alter the activity of the endogenous $\mathrm{GnRH}$ pulse generator. Recent studies have shown that a single bolus of kisspeptin influences the temporal pattern of release of subsequent $\mathrm{LH}$ pulses and that 
continuous exposure to exogenous kisspeptin increases the frequency and amplitude of LH pulses.

In one study a single intravenous bolus of KP-10 was administered and endogenous LH pulses measured [17]. KP10 induced (as expected) an acute LH rise and fall; however, it also appeared to reset the zero-point from which the periodicity of subsequent LH pulses is timed. The investigators detected a comparable interval between endogenous pulses and that following an artificially induced kisspeptin pulse, regardless of the time at which the kisspeptin was administered. This suggests that kisspeptin pulses may coordinate and direct the action of GnRH neurones, rather than simply modify their activity.

Since then, the effect of a single intravenous bolus of KP10 and of a single subcutaneous bolus of KP-54 on endogenous LH pulse generation in human females has been studied with some interesting results. It appears that not only does the response to kisspeptin demonstrate sexual dimorphism in humans, but also that $\mathrm{GnRH}$ pulse generation may be achieved or controlled by a different mechanism in males and females $[9,10]$.

The effects of a single subcutaneous bolus of KP-54 on LH pulses in healthy female volunteers $(n=6)$ has recently been reported [10]. This was done by measuring LH levels every ten minutes for four hours prior to a single subcutaneous injection of KP-54, in order to characterise the properties of endogenous pulses and then comparing this with the pattern of $\mathrm{LH}$ secretion observed in the four-hour period immediately after kisspeptin. Interestingly, in contrast to the effects observed in men using the intravenous route and using the KP-10 isoform, a subcutaneous bolus in women of KP-54 did not appear to delay the onset of the next LH pulse; rather, it shortened the interval between pulses. Investigators observed a higher number of LH pulses within the 4-hour-sampling period after injection, with most pulses observed following the highest dose studied $(0.6 \mathrm{nmol} / \mathrm{kg})$.

KP-10 given as a continuous intravenous infusion to healthy men and also recently to men with type two diabetes mellitus and hypotestosteronaemia has been shown to increase LH pulse frequency [7, 13]. Kisspeptin has also been shown in continuous intravenous infusion to restore pulsatile LH secretion in individuals with genetic dysfunction of the NKB signalling pathway, as previously discussed [15]. These findings suggest that it is not necessary for kisspeptin to be released in a pulsatile fashion and that $\mathrm{GnRH}$ neurons have autonomous pulse-generating activity which is induced under conditions of continuous exposure to kisspeptin.

More work is needed to outline the effects of the various kisspeptin isoforms, doses, and routes on the endogenous pattern of gonadotrophin secretion.

\section{Side Effects and Potential Action of Kisspeptin on Other Organ Systems}

It has been suggested that kisspeptin may mediate effects on several cell types out with the HPG axis. In vitro studies have shown that kisspeptin can mediate vasoconstriction [48] and inhibit angiogenesis [49], and it has been reported that kisspeptin stimulates glucose-induced insulin secretion in animal studies $[50,51]$. Healthy human volunteers have been closely monitored throughout kisspeptin infusion studies with regular heart rate and blood pressure measurement, and no symptoms or side effects have been demonstrated in studies of kisspeptin administration to humans. Elevated serum kisspeptin is not associated with an alteration in heart rate or blood pressure in humans [18]. No study of the effect of kisspeptin on glucose-induced insulin secretion in human has been published to date and this may be an area to be addressed in future work.

\section{Kisspeptin and Interaction with Other Neuroregulators of the Human Reproduction Axis}

Kisspeptin is an important neuropeptide exerting central control over GnRH neurons, but it is by no means alone in this task. GnRH pulsatility is the product of integration of multiple signals, and the number of neuropeptides linked to regulation of $\mathrm{GnRH}$ is becoming increasingly vast [52]. Those that seem to be of particular importance include members of the tachykinin peptide family neurokinin $B(\mathrm{NKB})$ and substance $\mathrm{p}$ (SP) and the neuropeptide dynorphin A (Dy). The regulatory network is complex, with transsynaptic inputs and glial cells playing a role. The neurotransmitters glutamate and noradrenaline mainly excite $\mathrm{GnRH}$ neurons, whilst GABA and endogenous opioids tend to inhibit [53].

The effect of NKB and Dy on GnRH release has previously attracted particular interest as a large proportion of the kisspeptin neurones immunohistochemically analysed for peptide expression in hypothalamic tissue sections from nonhuman mammals that have been noted to coexpress NKB and dynorphin. This has been referred to by some authors as the KNDy neuron $[54,55]$. Additionally, as previously described, neurokinin $\mathrm{B}$ is also the only other neuropeptide that has been shown, like kisspeptin, to be crucial to normal reproductive function in humans $[34,56]$.

Studies of the effect of NKB administration to mammals have not however produced results as consistent as those seen with kisspeptin. Whilst the data in animals does demonstrate that NKB can stimulate release of an LH pulse, it has been seen to have different effects in various species and acts in a complex fashion with a significant but as yet incompletely understood interaction with kisspeptin [57]. Recently, the first in man study of administration of NKB showed that at the doses tested NKB given as a $4 \mathrm{~h}$ or $8 \mathrm{~h}$ infusion had no demonstrable effect on the levels of gonadotrophins or sex steroids detectable in both healthy male and female volunteers [58].

Additionally, immunohistochemical analysis has recently been carried out on preparations of the infundibular/arcuate nucleus and stalk of three human males aged less than 37 years, and the neuroanatomy observed did not correlate with that expected from animal work [57]. The key findings from this small study were that (i) there were five times more detectable direct stimulatory synapses or contacts onto GnRH neurons arising from NKB expressing neurons than from KP expressing neurons; (ii) the pattern of coexpression 
of KP and NKB amongst neurons in these regions of the hypothalamus was not as expected from animal findings; not only $75 \%$ of KP neurons coexpressed NKB but also $33 \%$ of the total population of $\mathrm{NKB}$ expressing neurons coexpressed $\mathrm{KP}$ and there was therefore a large pool of NKB neurons that did not synthesize significant amounts of KP or dynorphin A; (iii) only very low levels of dynorphin immunoreactivity were detectable in these tissue preparations, and the fibres that did exist were generally distinct from KP neurons. Bearing in mind the fact that these were postmortem samples and therefore could have undergone degradation; it is worth considering that there may be some differences in the pattern of colocalization of these key neurotransmitters between rodents and humans. This is worthy of exploration in future. This will be a complicated task. There are a multitude of avenues in the neurobiology and physiology of kisspeptin that we have yet to explore, as has been highlighted in a recent review by Pinilla et al. [53].

\section{Developing Pharmacological Agents: Kisspeptin Receptor Antagonists and Kisspeptin Analogues}

There have been some notable advances in the last 5 years regarding the development of pharmacological agents that act on the kisspeptin system.

In 2009 , by a process of amino acid substitution within the KP-10 decapeptide sequence, a group of investigators produced a potent KISS1R antagonist (peptide 234) that blocked the effect of kisspeptin in mice, rats, ewes, and monkeys [59]. This antagonist was employed to provide direct evidence for many actions of kisspeptin, including that kisspeptin is essential for the generation of hypothalamic GnRH pulses and that kisspeptin is a target site for mediation of the negative feedback effects of gonadal sex steroids on the HPG axis. Its development also represents a significant platform on which to base development of analogues for use in humans.

Kisspeptin analogues that have high potency agonist activity have also been produced. Two molecules, TAK- 448 and TAK-683 (both synthetic nonapeptide analogues of the $\mathrm{KP}-10$ sequence), were used to suppress testosterone secretion in rodents in 2010 [60], and we have now seen the first phase 1 randomized double-blind placebo-controlled clinical trial of TAK-683 in healthy human males [61]. Subcutaneous infusion of TAK-683 for 2 weeks in healthy male volunteers has been demonstrated to induce rapid and effective reduction in serum testosterone, reversible upon discontinuation of the drug, in a dose-dependent fashion. This novel means of chemical castration could well be developed as a prostate cancer treatment in the near future.

\section{Conclusion}

In conclusion, recent studies of the effect of kisspeptin on reproductive hormone release in humans have furthered our understanding of the mechanisms underlying the hypothalamic control of the reproductive hormone axis. The data generated by the past decade's research has brought us a great deal closer to answering several questions which have eluded scientists and clinicians of endocrinology for many years, including the make-up of the endogenous GnRH pulse generator, the identity of the pubertal trigger, and the mechanism responsible for downregulation of the reproductive axis in times of stress such as negative energy balance or lactation. The discovery of kisspeptin may also offer therapeutic potential. The kisspeptin system has provided a new avenue to develop alternative treatments for disorders of reproduction characterized by low gonadotrophins or anovulation and also for development of high potency analogs akin to those already in place for $\mathrm{GnRH}$ that may be useful for conditions in which downregulation of reproductive activity is desirable. Indeed, kisspeptin analogs are already in development for such a purpose. We look forward with anticipation at seeing the developments in coming years.

\section{Conflict of Interests}

The authors declare that there is no conflict of interests regarding the publication of this paper.

\section{Acknowledgment}

Waljit S. Dhillo is funded by an NIHR Career Development Fellowship.

\section{References}

[1] M. Kotani, M. Detheux, A. Vandenbogaerde et al., "The metastasis suppressor gene KiSS-1 encodes kisspeptins, the natural ligands of the orphan G protein-coupled receptor GPR54," The Journal of Biological Chemistry, vol. 276, no. 37, pp.34631-34636, 2001.

[2] J. H. Lee, M. E. Miele, D. J. Hicks et al., "KiSS-1, a novel human malignant melanoma metastasis-suppressor gene," Journal of the National Cancer Institute, vol. 88, no. 23, pp. 1731-1737, 1996.

[3] T. Ohtaki, Y. Shintani, S. Honda et al., "Metastasis suppressor gene KiSS-1 encodes peptide ligand of a G-protein-coupled receptor," Nature, vol. 411, no. 6837, pp. 613-617, 2001.

[4] A. Abbara, R. Ratnasabapathy, C. N. Jayasena, and W. S. Dhillo, "The effects of kisspeptin on gonadotropin release in nonhuman mammals," Advances in Experimental Medicine and Biology, vol. 784, pp. 63-87, 2013.

[5] W. S. Dhillo, O. B. Chaudhri, M. Patterson et al., "Kisspeptin-54 stimulates the hypothalamic-pituitary gonadal axis in human males," Journal of Clinical Endocrinology and Metabolism, vol. 90, no. 12, pp. 6609-6615, 2005.

[6] C. N. Jayasena, G. M. K. Nijher, A. Abbara et al., "Twiceweekly administration of kisspeptin-54 for 8 weeks stimulates release of reproductive hormones in women with hypothalamic amenorrhea," Clinical Pharmacology and Therapeutics, vol. 88, no. 6, pp. 840-847, 2010.

[7] J. T. George, J. D. Veldhuis, A. K. Roseweir et al., "Kisspeptin10 is a potent stimulator of $\mathrm{LH}$ and increases pulse frequency in men," The Journal of Clinical Endocrinology and Metabolism, vol. 96, no. 8, pp. E1228-E1236, 2011.

[8] C. N. Jayasena, G. M. K. Nijher, A. N. Comninos et al., "The effects of kisspeptin-10 on reproductive hormone release show sexual dimorphism in humans," The Journal of Clinical Endocrinology \& Metabolism, vol. 96, no. 12, pp. E1963-E1972, 2011. 
[9] Y. M. Chan, J. P. Butler, V. F. Sidhoum, N. E. Pinnell, and S. B. Seminara, "Kisspeptin administration to women: a window into endogenous kisspeptin secretion and GnRH responsiveness across the menstrual cycle," Journal of Clinical Endocrinology and Metabolism, vol. 97, no. 8, pp. E1458-E1467, 2012.

[10] C. N. Jayasena, A. N. Comninos, J. D. Veldhuis et al., "A single injection of kisspeptin-54 temporarily increases luteinizing hormone pulsatility in healthy women," Clinical Endocrinology, vol. 79, no. 4, pp. 558-563, 2013.

[11] W. S. Dhillo, O. B. Chaudhri, E. L. Thompson et al., "Kisspeptin54 stimulates gonadotropin release most potently during the preovulatory phase of the menstrual cycle in women," Journal of Clinical Endocrinology and Metabolism, vol. 92, no. 10, pp. 39583966, 2007.

[12] C. N. Jayasena, G. M. K. Nijher, O. B. Chaudhri et al., "Subcutaneous injection of kisspeptin-54 acutely stimulates gonadotropin secretion in women with hypothalamic amenorrhea, but chronic administration causes tachyphylaxis," Journal of Clinical Endocrinology and Metabolism, vol. 94, no. 11, pp. 43154323, 2009.

[13] J. T. George, J. D. Veldhuis, M. Tena-Sempere, R. P. Millar, and R. A. Anderson, "Exploring the pathophysiology of hypogonadism in men with type 2 diabetes: Kisspeptin-10 stimulates serum testosterone and LH secretion in men with type 2 diabetes and mild biochemical hypogonadism," Clinical Endocrinology, vol. 79, no. 1, pp. 100-104, 2013.

[14] J. T. George, R. A. Anderson, and R. P. Millar, "Kisspeptin-10 stimulation of gonadotrophin secretion in women is modulated by sex steroid feedback," Human Reproduction, vol. 27, no. 12, pp. 3552-3559, 2012.

[15] J. Young, J. T. George, J. A. Tello et al., "Kisspeptin restores pulsatile LH secretion in patients with neurokinin B signaling deficiencies: physiological, pathophysiological and therapeutic implications," Neuroendocrinology, vol. 97, no. 2, pp. 193-202, 2013.

[16] C. N. Jayasena, A. N. Comninos, G. M. K. Nijher et al., "Twicedaily subcutaneous injection of kisspeptin-54 does not abolish menstrual cyclicity in healthy female volunteers," Journal of Clinical Endocrinology \& Metabolism, vol. 98, pp. 4464-4474, 2013.

[17] Y.-M. Chan, J. P. Butler, N. E. Pinnell et al., "Kisspeptin resets the hypothalamic GnRH clock in men," Journal of Clinical Endocrinology and Metabolism, vol. 96, no. 6, pp. E908-E915, 2011.

[18] G. M. K. Nijher, O. B. Chaudhri, R. Ramachandran et al., "The effects of kisspeptin-54 on blood pressure in humans and plasma kisspeptin concentrations in hypertensive diseases of pregnancy," The British Journal of Clinical Pharmacology, vol. 70, no. 5, pp. 674-681, 2010.

[19] M. L. Gottsch, M. J. Cunningham, J. T. Smith et al., "A role for kisspeptins in the regulation of gonadotropin secretion in the mouse," Endocrinology, vol. 145, no. 9, pp. 4073-4077, 2004.

[20] S. D. C. Bianco and U. B. Kaiser, "The genetic and molecular basis of idiopathic hypogonadotropic hypogonadism," Nature Reviews Endocrinology, vol. 5, no. 10, pp. 569-576, 2009.

[21] L. F. G. Silveira and A. C. Latronico, "Approach to the patient with hypogonadotropic hypogonadism," The Journal of Clinical Endocrinology \& Metabolism, vol. 98, no. 5, pp. 1781-1788, 2013.

[22] D. K. Lee, T. Nguyen, G. P. O'Neill et al., "Discovery of a receptor related to the galanin receptors," The FEBS Letters, vol. 446, no. 1, pp. 103-107, 1999.
[23] Y. Horikoshi, H. Matsumoto, Y. Takatsu et al., "Dramatic elevation of plasma metastin concentrations in human pregnancy: metastin as a novel placenta-derived hormone in humans," Journal of Clinical Endocrinology and Metabolism, vol. 88, no. 2, pp. 914-919, 2003.

[24] N. de Roux, E. Genin, J. Carel, F. Matsuda, J. Chaussain, and E. Milgrom, "Hypogonadotropic hypogonadism due to loss of function of the KiSS1-derived peptide receptor GPR54," Proceedings of the National Academy of Sciences of the United States of America, vol. 100, no. 19, pp. 10972-10976, 2003.

[25] S. B. Seminara, S. Messager, E. E. Chatzidaki et al., "The GPR54 gene as a regulator of puberty," The New England Journal of Medicine, vol. 349, no. 17, pp. 1614-1627, 2003.

[26] S. Funes, J. A. Hedrick, G. Vassileva et al., "The KiSS-1 receptor GPR54 is essential for the development of the murine reproductive system," Biochemical and Biophysical Research Communications, vol. 312, no. 4, pp. 1357-1363, 2003.

[27] L. G. Silveira, A. C. Latronico, and S. B. Seminara, "Kisspeptin and clinical disorders.," Advances in Experimental Medicine and Biology, vol. 784, pp. 187-199, 2013.

[28] M. G. Teles, E. B. Trarbach, S. D. Noel et al., "A novel homozygous splice acceptor site mutation of KISS1R in two siblings with normosmic isolated hypogonadotropic hypogonadism," European Journal of Endocrinology, vol. 163, no. 1, pp. 29-34, 2010.

[29] A. K. Topaloglu, J. A. Tello, L. D. Kotan et al., "Inactivating KISS1 mutation and hypogonadotropic hypogonadism," The New England Journal of Medicine, vol. 366, no. 7, pp. 629-635, 2012.

[30] J. R. Pedersen-White, L. P. Chorich, D. P. Bick, R. J. Sherins, and L. C. Layman, "The prevalence of intragenic deletions in patients with idiopathic hypogonadotropic hypogonadism and Kallmann syndrome," Molecular Human Reproduction, vol. 14, no. 6, pp. 367-370, 2008.

[31] M. G. Teles, S. D. C. Bianco, V. N. Brito et al., "A GPR54-activating mutation in a patient with central precocious puberty," The New England Journal of Medicine, vol. 358, no. 7, pp. 709715, 2008.

[32] J. Roa, E. Vigo, J. M. Castellano et al., "Hypothalamic expression of KiSS-1 system and gonadotropin-releasing effects of kisspeptin in different reproductive states of the female rat," Endocrinology, vol. 147, no. 6, pp. 2864-2878, 2006.

[33] J. T. Smith, "Sex steroid control of hypothalamic Kiss1 expression in sheep and rodents: comparative aspects," Peptides, vol. 30, no. 1, pp. 94-102, 2009.

[34] A. K. Topaloglu, F. Reimann, M. Guclu et al., "TAC3 and TACR3 mutations in familial hypogonadotropic hypogonadism reveal a key role for Neurokinin B in the central control of reproduction," Nature Genetics, vol. 41, no. 3, pp. 354-358, 2009.

[35] P. Limonta and M. Manea, "Gonadotropin-releasing hormone receptors as molecular therapeutic targets in prostate cancer: current options and emerging strategies," Cancer Treatment Reviews, vol. 39, no. 6, pp. 647-663, 2013.

[36] S. B. Seminara, M. J. DiPietro, S. Ramaswamy, W. F. Crowley Jr., and T. M. Plant, "Continuous human metastin 45-54 infusion desensitizes $\mathrm{G}$ protein-coupled receptor 54-induced gonadotropin-releasing hormone release monitored indirectly in the juvenile male Rhesus monkey (Macaca mulatta): a finding with therapeutic implications," Endocrinology, vol. 147, no. 5, pp. 2122-2126, 2006.

[37] S. Ramaswamy, S. B. Seminara, C. R. Pohl, M. J. Dipietro, W. F. Crowley Jr., and T. M. Plant, "Effect of continuous intravenous 
administration of human metastin $45-54$ on the neuroendocrine activity of the hypothalamic-pituitary-testicular axis in the adult male rhesus monkey (Macaca mulatta)," Endocrinology, vol. 148, no. 7, pp. 3364-3370, 2007.

[38] E. L. Thompson, V. Amber, G. W. H. Stamp et al., "Kisspeptin54 at high doses acutely induces testicular degeneration in adult male rats via central mechanisms," British Journal of Pharmacology, vol. 156, no. 4, pp. 609-625, 2009.

[39] E. L. Thompson, K. G. Murphy, M. Patterson et al., "Chronic subcutaneous administration of kisspeptin-54 causes testicular degeneration in adult male rats," American Journal of Physiology-Endocrinology and Metabolism, vol. 291, no. 5, pp. E1074-E1082, 2006.

[40] V. M. Navarro, R. Fernández-Fernández, J. M. Castellano et al., "Advanced vaginal opening and precocious activation of the reproductive axis by KiSS-1 peptide, the endogenous ligand of GPR54," The Journal of Physiology, vol. 561, no. 2, pp. 379-386, 2004.

[41] T. M. Plant, S. Ramaswamy, and M. J. DiPietro, "Repetitive activation of hypothalamic G protein-coupled receptor 54 with intravenous pulses of kisspeptin in the juvenile monkey (Macaca mulatta) elicits a sustained train of gonadotropinreleasing hormone discharges," Endocrinology, vol. 147, no. 2, pp. 1007-1013, 2006.

[42] J. M. Castellano, V. M. Navarro, R. Fernández-Fernández et al., "Changes in hypothalamic KiSS-1 system and restoration of pubertal activation of the reproductive axis by kisspeptin in undernutrition," Endocrinology, vol. 146, no. 9, pp. 3917-3925, 2005.

[43] C. N. Jayasena, A. Abbara, J. D. Veldhuis et al., "Increasing LH pulsatility in women with hypothalamic amenorrhoea using intravenous infusion of kisspeptin-54," Journal of Clinical Endocrinology and Metabolism, vol. 99, no. 6, pp. E953-E961, 2014.

[44] Y.-M. Chan, "Effects of kisspeptin on hormone secretion in humans," Advances in Experimental Medicine and Biology, vol. 784, pp. 89-112, 2013.

[45] E. Hrabovszky and Z. Liposits, "Afferent neuronal control of type-I gonadotropin releasing hormone neurons in the human," Frontiers in Endocrinology, vol. 4, article 130, 2013.

[46] X. F. Li, J. S. Kinsey-Jones, Y. Cheng et al., "Kisspeptin signalling in the hypothalamic arcuate nucleus regulates $\mathrm{GnRH}$ pulse generator frequency in the rat," PLoS ONE, vol. 4, no. 12, Article ID e8334, 2009.

[47] B. Francou, J. Bouligand, A. Voican et al., "Normosmic congenital hypogonadotropic hypogonadism due to TAC3/TACR3 mutations: characterization of neuroendocrine phenotypes and novel mutations," PLoS ONE, vol. 6, no. 10, Article ID e25614, 2011.

[48] I. Sawyer, S. Smillie, J. V. Bodkin, E. Fernandes, K. T. O’Byrne, and S. D. Brain, "The vasoactive potential of kisspeptin-10 in the peripheral vasculature," PLoS ONE, vol. 6, no. 2, Article ID e14671, 2011.

[49] T. Ramaesh, J. J. Logie, A. K. Roseweir et al., "Kisspeptin-10 inhibits angiogenesis in human placental vessels ex vivo and endothelial cells in vitro," Endocrinology, vol. 151, no. 12, pp. 59275934, 2010.

[50] A. C. Hauge-Evans, C. C. Richardson, H. M. Milne, M. R. Christie, S. J. Persaud, and P. M. Jones, "A role for kisspeptin in islet function," Diabetologia, vol. 49, no. 9, pp. 2131-2135, 2006.

[51] J. E. Bowe, A. J. King, J. S. Kinsey-Jones et al., "Kisspeptin stimulation of insulin secretion: mechanisms of action in mouse islets and rats," Diabetologia, vol. 52, no. 5, pp. 855-862, 2009.
[52] R. A. Steiner, "Kisspeptin: past, present, and prologue," Advances in Experimental Medicine and Biology, vol. 784, pp. 3-7, 2013.

[53] L. Pinilla, E. Aguilar, C. Dieguez, R. P. Millar, and M. TenaSempere, "Kisspeptins and reproduction: physiological roles and regulatory mechanisms," Physiological Reviews, vol. 92, no. 3, pp. 1235-1316, 2012.

[54] G. Cheng, L. M. Coolen, V. Padmanabhan, R. L. Goodman, and M. N. Lehman, "The kisspeptin/neurokinin B/dynorphin (KNDy) cell population of the arcuate nucleus: sex differences and effects of prenatal testosterone in sheep," Endocrinology, vol. 151, no. 1, pp. 301-311, 2010.

[55] M. N. Lehman, L. M. Coolen, and R. L. Goodman, "Minireview: kisspeptin/neurokinin B/dynorphin (KNDy) cells of the arcuate nucleus: a central node in the control of gonadotropin-releasing hormone secretion," Endocrinology, vol. 151, no. 8, pp. 34793489, 2010.

[56] J. Young, J. Bouligand, B. Francou et al., "TAC3 and TACR3 defects cause hypothalamic congenital hypogonadotropic hypogonadism in humans," The Journal of Clinical Endocrinology \& Metabolism, vol. 95, no. 5, pp. 2287-2295, 2010.

[57] E. Hrabovszky, M. T. Sipos, C. S. Molnár et al., "Low degree of overlap between kisspeptin, neurokinin B, and dynorphin immunoreactivities in the infundibular nucleus of young male human subjects challenges the KNDy neuron concept," Endocrinology, vol. 153, no. 10, pp. 4978-4989, 2012.

[58] C. N. Jayasena, A. N. Comninos, A. de Silva et al., "Effects of neurokinin B administration on reproductive hormone secretion in healthy men and women," Journal of Clinical Endocrinology and Metabolism, vol. 99, no. 1, pp. E19-E27, 2014.

[59] A. K. Roseweir, A. S. Kauffman, J. T. Smith et al., "Discovery of potent kisspeptin antagonists delineate physiological mechanisms of gonadotropin regulation," Journal of Neuroscience, vol. 29, no. 12, pp. 3920-3929, 2009.

[60] H. Matsui, Y. Takatsu, A. Tanaka et al., "251 potent and efficient testosterone suppression by chronic administration of novel metastin analogues, TAK-448 and TAK-683, in male rats," European Journal of Cancer Supplements, vol. 8, article 66, 2010.

[61] G. Scott, I. Ahmad, K. Howard et al., "Double-blind, randomized, placebo-controlled study of safety, tolerability, pharmacokinetics and pharmacodynamics of TAK-683, an investigational metastin analogue in healthy men," British Journal of Clinical Pharmacology, vol. 75, no. 2, pp. 381-391, 2013. 

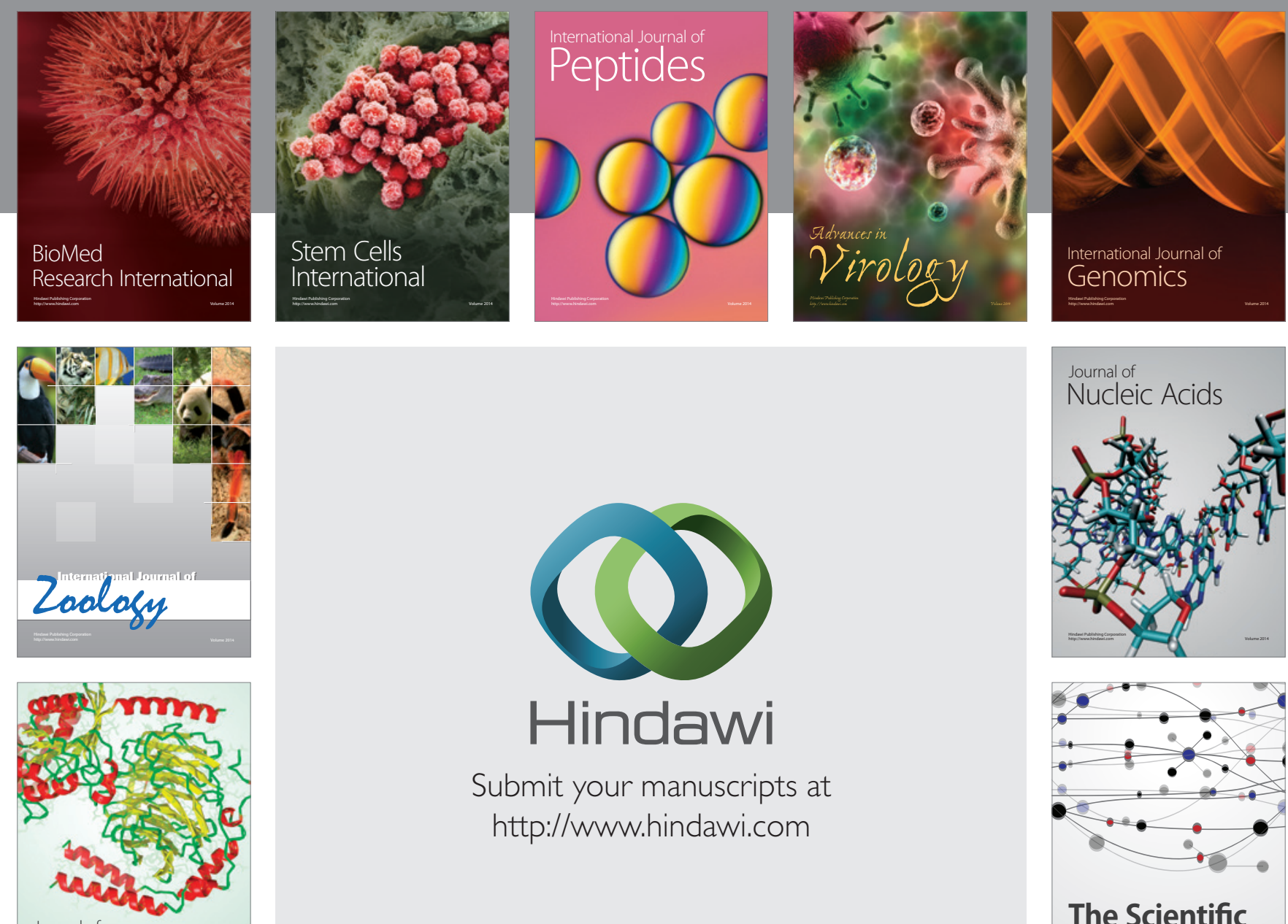

Submit your manuscripts at

http://www.hindawi.com

Journal of
Signal Transduction
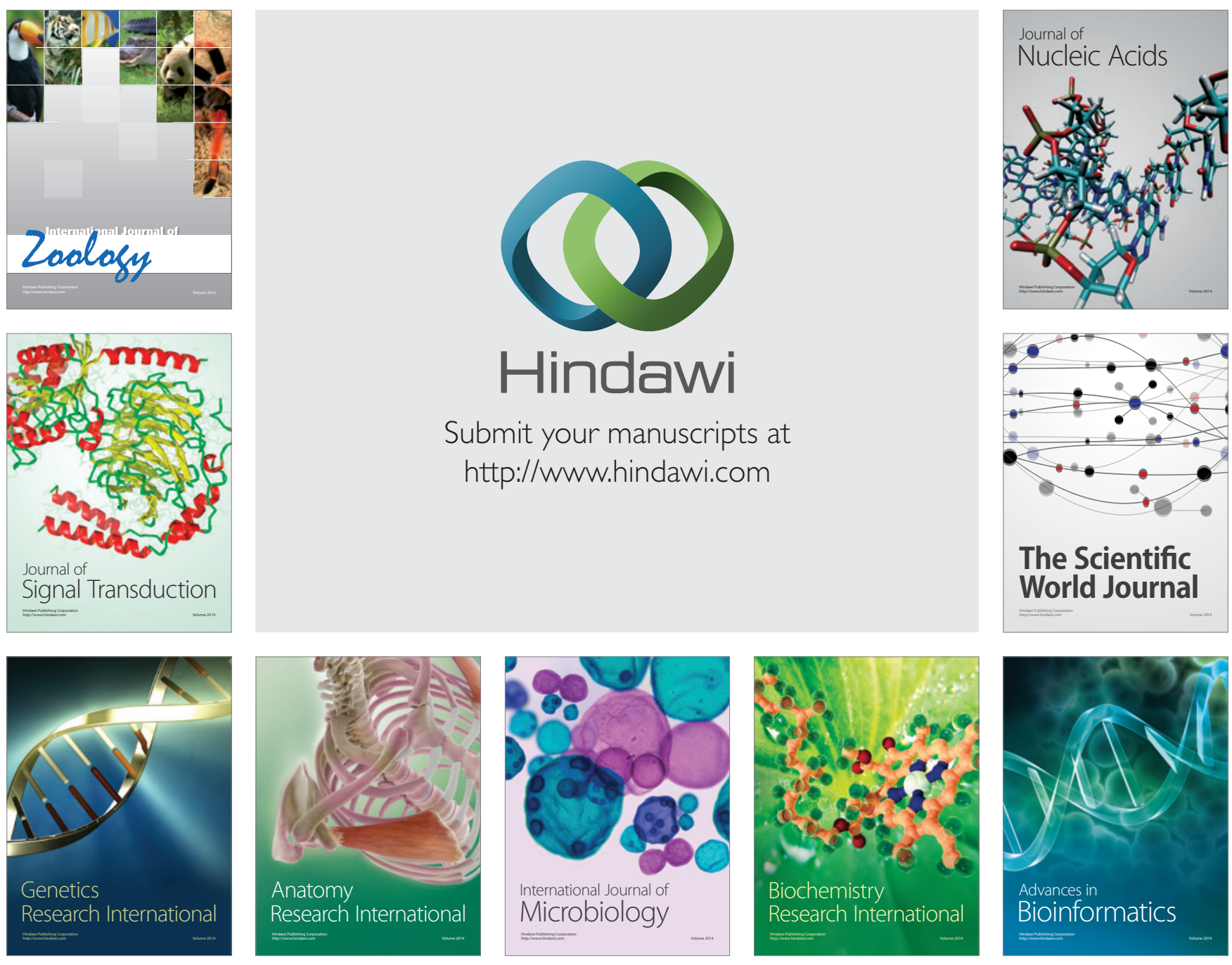

The Scientific World Journal
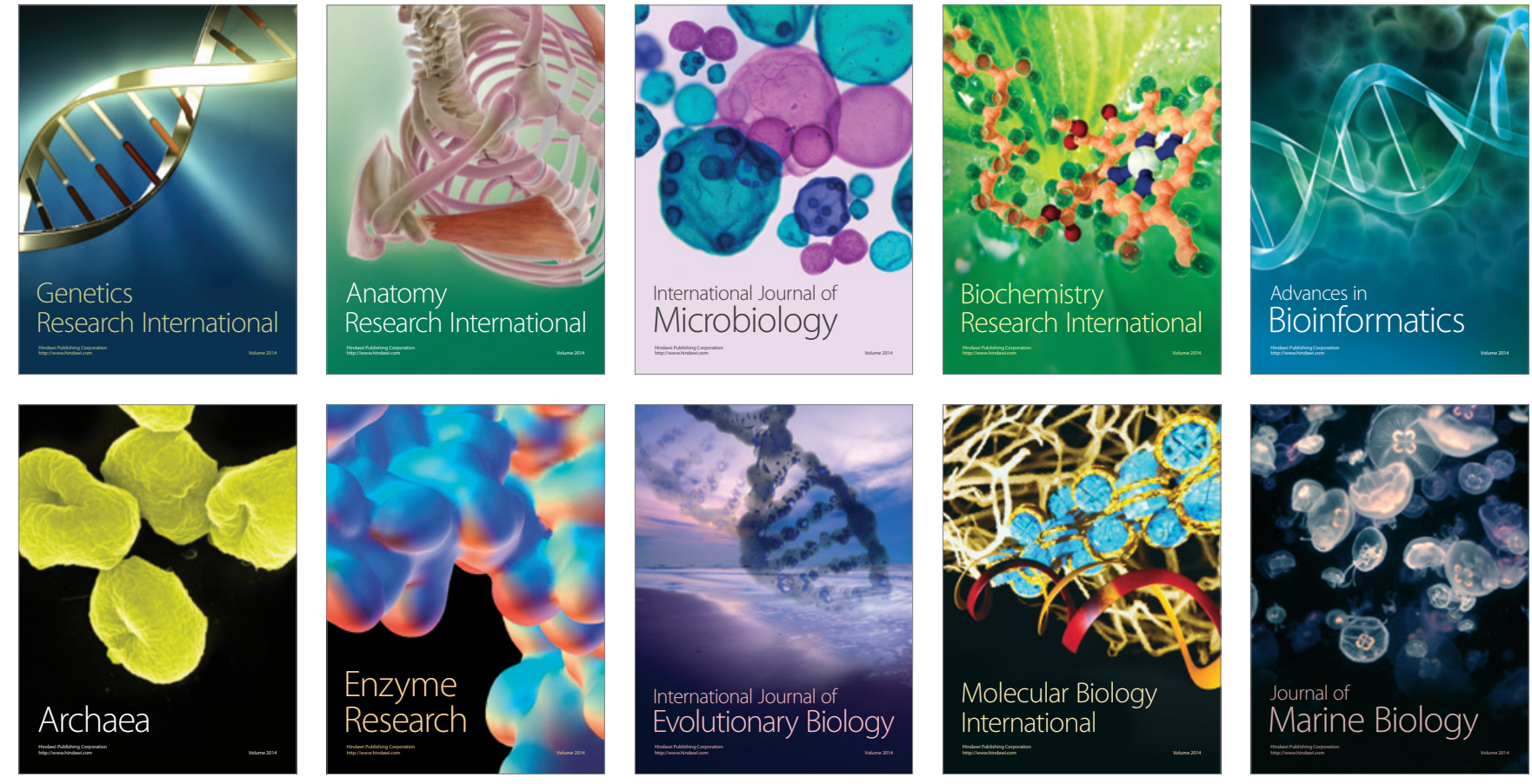